\title{
marges Marges
}

revue d'art contemporain Revue d'art contemporain

15 | 2012

Démocratiser l'art [contemporain]

\section{Co-dépendance et recyclage hollywoodien de l' Indie Spirit : une redéfinition problématique du cinéma indépendant américain contemporain}

\section{Célia Sauvage}

\section{OpenEdition}

Journals

Édition électronique

URL : http://journals.openedition.org/marges/359

DOI : 10.4000/marges.359

ISSN : 2416-8742

Éditeur

Presses universitaires de Vincennes

\section{Édition imprimée}

Date de publication : 15 octobre 2012

Pagination : 96-106

ISBN : 978-2-84292-354-9

ISSN : 1767-7114

Référence électronique

Célia Sauvage, « Co-dépendance et recyclage hollywoodien de l'Indie Spirit : une redéfinition problématique du cinéma indépendant américain contemporain », Marges [En ligne], 15 | 2012, mis en ligne le 15 octobre 2013, consulté le 01 mai 2019. URL : http://journals.openedition.org/marges/359 ; DOI : 10.4000/marges.359 


\section{Co-dépendance et recyclage hollywoodien de l'Indie Spirit: une redéfinition problématique du cinéma indépendant américain contemporain}

On peut débattre pour savoir s'il existe un authentique cinéma indépendant américain. Cependant, il est devenu difficile de nier l'existence de l'étiquette de "cinéma indépendant américain », qui non seulement existe bel et bien mais qui est également parfaitement intégrée dans le discours critique et spectatoriel. "Film indépendant » signifie tout à la fois un mode de production, une forme esthétique, une position idéologique, ainsi qu'un mode de consommation et d'appréciation des films contemporains. Ce terme renvoie au débat traditionnel entre l'art et le commerce, opposant d'un côté une position auctoriale et de l'autre une position plus mercantile visant le profit économique. Dans cette perspective, le cinéma indépendant est associé à une vision idéaliste de la production cinématographique, selon 
laquelle il serait possible de créer un film de manière complètement autonome, sans interférence et sans pression du système dominant hollywoodien. Une telle position est-elle envisageable alors même qu'Hollywood se lance également dans le commerce de l'«auteurisme » et que la production à grande échelle de films indépendants a débouché sur le concept d'indiewood?

\section{L'Indiewood, un système économique complexe, entre indépendance et co-dépendance}

Traditionnellement, l'industrie cinématographique, et plus particulièrement américaine, est divisée en deux pôles opposés. D'un côté, le cinéma hollywoodien grand public (mainstream), secteur dominant, dont les blockbusters, films à gros budget, représentent la forme la plus aboutie. De l'autre, le cinéma indépendant, strictement distinct du système hollywoodien, secteur hétérogène et vaste qui peut aller des films expérimentaux, avant-gardistes, aux petites productions, voire à des films sans budget. Greg Merritt propose ainsi, dans son ouvrage, Celluloid Mavericks: A History of American Independent Films, deux définitions du cinéma indépendant. Selon lui, « tout film financé et produit de manière complètement autonome, indépendamment de tout studio, peu importe leur taille » peut être considéré comme un film indépendant. Mais il y associe également «l'idée communément admise selon laquelle l'indépendance est caractérisée, non par un mode de financement, mais par un esprit (indie spirit), qui offre une vision alternative/ $\mathbf{1}$ ॥. L'indépendance ou l'autonomie des films se définirait ainsi selon trois critères. Tout d'abord, leur place dans le secteur industriel - s'ils appartiennent ou non à un studio d'Hollywood). Deuxièmement, leurs choix esthétiques et formels (en rupture ou non avec les mêmes conventions hollywoodiennes). Troisièmement, leur position vis-à-vis d'une idéologie politique, sociale, culturelle.

Cependant, le succès grandissant des films indépendants américains a amené une restructuration institutionnelle considérable du secteur. Aujourd'hui, la base de celui-ci est organisée autour des filières spécialisées de grands studios (specialty divisions), privilégiant le développement de films plus originaux, plus ambitieux, mais souvent moins porteurs financièrement. Ainsi, de nombreux mini-studios indépendants ont été rachetés par des majors américaines, comme ce fut le cas de Miramax, racheté par Disney en 1993. D'autres ont vu le jour grâce au financement direct d'une major. Par exemple, les deux filières spécialisées les plus reconnues, Focus Features et Fox Searchlight, sont rattachées respectivement à Universal et à la Fox. Ces filières spécialisées ont généralement un degré d'autonomie suffisant
/1 Greg Merritt, Celluloid Mavericks: A History of American Independent Films, New York, Da Capo Press, 1999, p. XII. Toutes les traductions sont de l'auteur, sauf mention contraire. 
/2 Peter Biskind suggère que le terme est apparu en 1994, dans son ouvrage, Sexe, Mensonge et Hollywood (2004) alors que Geoff King l'attribue véritablement à un article écrit par la réalisatrice indépendante, Sarah Jacobson, « Understanding D.I.Y », indieWIRE,

novembre 1997.

/3 Geoff King, Indiewood, USA: Where Hollywood Meets Independent Cinema, Londres, I.B. Tauris, 2009, p. 2.

/4 Anthony Kaufman, Steven Soderbergh: Interviews, Jackson, University Press of Mississippi, p. xvı.

/5 James Schamus, " To the Rear of the Back End: The Economics of Independent Cinema », dans Neale et Smith (sld), Contemporary Hollywood Cinema, London \& New York, Routledge, 1998, p. 103.

/6 E. Deidre Pribram, Cinema and Culture: Independent Film in the United States, 1980-2001, New York, Peter Lang Publishing, 2002, p. 3.

/7 Lynn Hirschberg, "The Two Hollywoods ", New York Times, 16 novembre 1997 , [http:// www.nytimes. com/1997/11/16/ magazine/the-twohollywoods.html]. pour décider de produire ou de distribuer des films sans l'accord du studio auquel elles appartiennent. L'avantage néanmoins pour les studios n'est pas négligeable. Ces filières partagent notamment avec les studios le succès financier occasionnel de leurs films. Ces derniers peuvent également espérer découvrir de nouveaux talents à réemployer dans des films à plus grand budget. Ils gagnent également une certaine crédibilité artistique à apposer leurs noms à des films plus indépendants, notamment lorsque ceux-ci sont récompensés par une reconnaissance critique et parfois couronnés d'Oscars.

En réalité, les films appartiennent aujourd'hui à un secteur hybride, communément appelé l'indiewood aux États-Unis, terme apparu au milieu des années 1990/2. Cette branche récente du secteur indépendant annonce de nouvelles formes de pratique de cette indépendance, plus institutionnalisées. En introduction de son ouvrage Indiewood, USA: Where Hollywood Meets Independent Cinema, Geoff King définit la notion ainsi : "Une caractéristique centrale du cinéma de l'Indiewood [...] est le mélange, à l'œuvre dans les films, à la fois de conventions mainstream dominantes et de marqueurs de distinction ciblant une niche spectatorielle spécifique/3. ". Dans cette même perspective, Anthony Kaufman parle d'un « mélange d'autonomie et d'individualité avec une machine marketing hollywoodienne et un star system/4. ». Victime de son succès, le secteur indépendant s'est constitué ainsi comme un nouveau secteur industrialisé, institutionnalisé, " un succès qui fait ressembler de plus en plus les films indépendants à un microcosme digne d'un business de studio ", comme l'écrit James Schamus/5. En s'organisant comme un studio hollywoodien, avec son star system, sa machine marketing et sa niche spectatorielle, le secteur indépendant ne peut plus être considéré comme alternatif, à la marge d'Hollywood, mais plutôt comme un secteur central dans l'offre américaine. Au point que certains, comme E. Deidre Pribram, s’interrogent pour savoir si dans les faits l'indiewood n'est pas une manière « de désigner un nouvel Hollywood/6 ». Dès 1997, avant même l'apparition de cette nouvelle désignation, le New York Times consacrait déjà un numéro spécial à l'essor du secteur indépendant, sous le titre de "Two Hollywoods »: "Les spectateurs ont maintenant le choix entre les produits de deux marchés du film distincts, chacun avec sa propre sensibilité, son modèle économique, sa galerie de personnages et d'acteurs, son mode de vie. L'un est dédié au marché des blockbusters aux budgets se chiffrant à plusieurs millions de dollars et aux stars multi-récompensées. Alors que le second défend les notions de liberté artistique, le style guerilla et les idées risquées/7. ".

Les films dits indépendants ne s'inscrivent plus strictement comme alternative au secteur mainstream, ils évoluent au sein de ce secteur 
industriel. Les frontières entre les deux secteurs deviennent de plus en plus floues. Chacun emprunte à l'autre de nouvelles formes et caractéristiques pour se redéfinir. C'est notamment le recyclage de l'esthétique petit budget et de l'«auteurisme » par Hollywood qui force le secteur indépendant à se redéfinir autrement.

\section{L'Indie Spirit recyclé par Hollywood}

Même si la définition économique du secteur reste problématique, les films indépendants américains sont devenus « un euphémisme pour désigner des productions à petits budgets/8 », selon Emanuel Levy. Il reconnaît d'ailleurs que bon nombre de producteurs exécutifs de studios indépendants mentent régulièrement sur le budget réellement dépensé en présentant une version plus "low-budget». Le dernier exemple en date est Monsters de Gareth Edwards en 2010, un petit film indépendant qui avait axé toute sa promotion sur la débrouillardise de son réalisateur, capable de faire un film de monstres avec « un budget de seulement 15000,00 dollars alors qu'il a l'air 700 fois plus cher/9 ". Le mythe du budget de seulement 15000,00 dollars s'est propagé sur Internet avant d'être corrigé et finalement évalué à près de 800000,00 dollars. Cette somme, qui reste dérisoire comparée aux budgets des blockbusters hollywoodiens, est néanmoins beaucoup plus importante que celle annoncée à l'origine, prouvant la valeur accordée, dans le milieu indépendant, au fait de défendre un petit budget, quitte à mentir. Le réalisateur continue d'ailleurs à entretenir le flou, évitant savamment les questions lorsqu'on lui demande le véritable budget de son film: "Honnêtement, je ne peux pas vous dire. La raison principale, c'est que je ne le sais pas moi-même, et si je le savais, je continuerai à ne pas le dire. [rires] Mais c'est vraiment un petit, petit, petit, petit budget. Un micro-budget/10. ". Avant Monsters, bon nombre de films indépendants ont fait leur renommée grâce à leur petit budget, que ce soit El Mariachi de Robert Rodriguez en 1992 (7 ooo dollars), Clerks de Kevin Smith en 1994 (27 000,00 dollars) ou le cas le plus connu, Le Projet Blair Witch de Daniel Myrick et Eduardo Sánchez en 1999 (25000,00 dollars). L'occultation du budget réel pour entretenir le mythe du petit budget continue de faire ses preuves encore aujourd'hui. Alors que l'un des deux réalisateurs du Projet Blair Witch, Eduardo Sánchez, tente de faire parler de son nouveau film, il persiste dans cette lancée et refuse de dévoiler son budget en entretien, préférant symboliquement le comparer à un blockbuster hollywoodien. Il déclare ainsi que son film a coûté « beaucoup moins que Spider-Man. Probablement même moins cher que le budget restauration du tournage de Spider Man. C'était rien, mec/11. ».
/8 Emanuel Levy, Cinema of Outsiders: The Rise of American Independent Film, New York, New York University Press, p. 505.

/9 Peter Sciretta, " How Gareth Edwards Shot Monsters on an Incredibly Low Budget ", Slashfilm, 3 juin 2010, [http://www. slashfilm. com/how-garethedwards-shot-monsterson-an-incredibly-lowbudget/].

/10 Cole Abaius, « Interview: Monsters' Director Gareth Edwards On Pushing His Actors, Delays Caused by Prison Riots, and Streching a Micro-Budget », Film School Rejects, 28 octobre 2010, [http:// www.filmschoolrejects. com/features/interviewmonsters-director-garethedwards-on-pushing-hisactors-delays-caused-byprison-riots-andstretching-a-microbudget. php].

/11 Eric Kohn, « Interview: How Blair Witch Co-Director Eduardo Sanchez Got His Groove Back with Lovely Molly ", IndieWire, 16 septembre 2011, [http://www. indiewire.com/article/ interview_how_blair_ witch_co-director_ eduardo_sanchez_got_ his_gro ove_bac k_w]. 
/12 Emanuel Levy, op. cit., p. 194.

/13 Eric Kohn, « Making Movies with Laptops and Ingenuity ", New York Times, 13 octobre 2010, [http://www.nytimes. com/2010/10/17/ movies/17monsters.html].

/14 ibid.

/15 Steven Zeitchick et Amy Kaufman, «The Devil Inside is the Latest to Use Found Footage ", Los Angeles Times, 10 janvier 2012, [http://articles. latimes.com/2012/ jan/10/entertainment/ la-et-foundfootage-20120110].

/16 David Bordwell, « Unsteadycam chronicles", davidbordwell.net, 17 août 2007, [http:// www.davidbordwell.net/ blog/2007/08/17/ unsteadicam-chronicles/].
«Le budget est devenu l'esthétique/12 » conclut donc Emanuel Levy, en citant le réalisateur indépendant Hal Hartley. Effectivement, associer symboliquement les deux respecte la logique de l'image de marque construite par le cinéma indépendant américain. Motiver un choix esthétique par un budget serré permet de défendre l'idée d'artisanat et de création indépendante, chère à ce secteur du cinéma américain. Ceci contribue à l'opposition symbolique au cinéma hollywoodien mainstream : l'opposition à des films à gros budget, à effets spéciaux, comme le suggère le co-réalisateur du Projet Blair Witch. Respectant cette logique, le film Monsters était lui-même présenté comme " un contre-exemple radical face aux productions hollywoodiennes coûteuses/13 ", le critique insistant sur les 250 plans à effets spéciaux, générés par "une armée constituée uniquement d'une personne/14 ». Pour le cinéma indépendant, défendre l'artisanat s'exprime non seulement par une logique presque mensongère visant à réduire le budget des films dans le discours public, mais également par une logique visuelle inverse à celle défendue par les blockbusters américains. Contrairement à ce qui fait la force d'un film comme Monsters qui tente de rivaliser sur le plan des effets spéciaux avec Hollywood, la plupart des films indépendants optent au contraire pour une logique a priori d'anti-esthétisme, de salissement de l'image, d'amateurisme avoué parfois même, à l'image du Projet Blair Witch tourné en vidéo par trois étudiants en cinéma partis en reportage. Cet amateurisme de l'image, allant à l'encontre des canons hollywoodiens d'une belle image, produit une impression de réalisme, de «vérité low-budget/15 ». Ce rapport plus direct, plus intime, à la réalité a évidemment vite été recyclé par Hollywood, tenté à son tour par un processus d'authentification de son cinéma.

Ainsi sont apparus des films tournés caméra à l'épaule, principalement des films d'action et des films d'horreur. Ce nouveau style, appelé tantôt shaky cam, run-and-gun style, ou panic cam, combine un pseudo-amateurisme, porté par une caméra tremblante créant une illusion de réalisme et d'immédiateté de l'expérience, qui va à l'encontre de l'hyperfluidité des images en mouvement d'ordinaire cadrées au steadycam. Ceci amène David Bordwell à renommer d'ailleurs ces nouveaux films des « unsteadycam chronicles/16 ». Cette impression de caméra instable est souvent renforcée par les personnages qui deviennent eux-mêmes les chefs opérateurs, comme dans Cloverfield de Matt Reeves en 2008, ou encore Paranormal Activity d'Oren Peli en 2007. Ce mode d'identification de la vision à la première personne renforce l'impression d'intimité avec les personnages. Matt Reeves déclare ainsi à propos de Cloverfield: "Ce film est centré sur les gens ordinaires et je voulais que les spectateurs puissent s'identi- 
fier aux personnages et se dire qu'à leur place, avec une caméra, eux aussi auraient pu faire ce film/17. ".

Filmant à la première personne, leur stress et leur peur deviennent communicatifs et contaminent non seulement les spectateurs mais également l'image, d'où la caméra le plus souvent tremblante, les cadrages et les mises au point approximatifs. Malgré le budget faramineux de ces productions hollywoodiennes, le manque de maîtrise technique des personnages permet de justifier une image proche de l'amateurisme, une image salie et graineuse, une image low-fi de basse qualité. Ainsi, une caméra qui continue à tourner en filmant le sol, voire qui tombe à terre, lorsqu'un personnage se fait attaquer par un monstre, dans Cloverfield, ne passe pas pour un défaut technique. Cette technique était déjà présente dans Le Projet Blair Witch, si l'on se souvient du tout dernier plan du film avec la caméra abandonnée à même le sol au moment où le personnage-opérateur disparaît mystérieusement hors-champ. Les images d'ordinaire coupées au montage sont ici conservées pour contribuer au contraire à l'illusion de réalité et d'immédiateté voulue par ce nouveau genre des shaky cam. Les images sont sales et non professionnelles, elles doivent donc dire la vérité. Elles sont authentiques.

Le recyclage de l'esthétique petit budget, d'ordinaire fer de lance de la production indépendante, permet symboliquement d'apporter un cachet de vérité, d'authentifier et de légitimer le cinéma hollywoodien, dont le succès repose pourtant d'ordinaire sur la technicité affichée des films, rivalisant de technologies nouvelles et d'effets visuels époustouflants. Cette ambivalence se retrouve dans le discours de Matt Reeves, le réalisateur de Cloverfield, qui déclare ainsi à propos du tournage de son film: "C'est incroyable d'arriver à un tel résultat avec une handy-cam. Ca m'a convaincu de tourner l'essentiel du film en handy-cam. Cependant les responsables des effets spéciaux m'ont expliqué qu'il faudrait également une caméra d'une meilleure définition. [...] II fallait que [les techniciens] filment avec ces grosses caméras professionnelles qui pesaient 20 ou $30 \mathrm{~kg}$ comme si elles n'avaient pesé que quelques centaines de grammes. [...] J'ai tenu à tourner moi-même quelques scènes car je voulais apporter cette petite touche amateur, faire comme si nos plans soigneusement préparés étaient totalement aléatoires/18. ". Si l'on s’interroge alors sur le réel intérêt pour les productions hollywoodiennes de recycler ce procédé de l'amateurisme des images, l'intérêt économique et budgétaire est vite balayé. En effet, les budgets des films ne diminuent guère. Comme le réalisateur de Cloverfield l'avoue, l'amateurisme n'est qu'une illusion qui doit masquer le budget réel du film. Le film est estimé à près de 25 millions de dollars alors que Le Projet Blair
/17 Matt Reeves, interviewé pour Premiere. fr, [http://www.premiere. $\mathrm{fr} /$ Cinema/Excluscinema/Interviewcinema/Matt-Reeves-lerealisateur-de-Cloverfielden-interview].

/18 Matt Reeves, ibid. 
/19 Anne Thompson, "Greengrass brings auds in picture ", Variety, 3 août 2007, [http:// www.variety.com/article/ VR1117969675?ref Catld $=25$ 08].

\section{/ 20 La Mémoire dans} la peau (2002), La Mort dans la peau (2004), La Vengeance dans la peau (2007).

/21 David Bordwell, op. cit.

/ 22 Roger Ebert, "Shake, Rattle, and Bourne! », rogerebert.com, 16 août 2007, [http: //rogerebert. suntimes.com/apps/ pbcs.dll/ article?AID=/20070816/ COMMENT ARY/708 16001].

/23 Thomson Anne, op. cit.

\section{/24 ibid.}

Witch n'avait coûté qu'à peine 25000,00 dollars. Dans un article de Variety, le style de Paul Greengrass, le réalisateur de La Vengeance dans la peau (2007), est qualifié "de cinéma intime au service d'un budget de 130 millions de dollars/19 ", formule soulignant tout le paradoxe entre le budget affiché et celui réellement dépensé.

Finalement, l'intérêt du run-and-gun style n'est pas la qualité de l'image, faussement salie, mais la mise en mouvement de la caméra et la vision à la première personne. Ce nouveau type d'image-action, en mouvement constant, peut apparaître comme un sensationnalisme visuel mais il s'inscrit dans le fond parfaitement dans les procédés d'immersion maximale mis en œuvre par les films hollywoodiens contemporains. La trilogie de Jason Bourne/20 a notamment popularisé, voire caricaturé selon les plus critiques, cette caméra en mouvement constant. David Bordwell rappelle qu'en effet « La Vengeance dans la peau est rapide, avec 3200 plans en l'espace de 105 minutes, ce qui fait une moyenne de deux secondes par plan/21». Cependant cette immersion excessive a un prix. Le rythme frénétique de l'action cumulé à la vitesse de coupe, aux nombreux effets de zoom, dézoom, filé optique, peut provoquer un sentiment d'instabilité qui entame la résistance physique des spectateurs. Il arrive que ces derniers soient pris d'étourdissement, de maux de tête, de désorientation, comme le rapporte Roger Ebert après avoir reçu de nombreux messages de spectateurs/22. Cet inconvénient mis à part, l'avantage d'un mouvement constant participe à l'impression d'action immédiate, non contrôlée, d'un flux d'énergie constant. Dans cette perspective, Paul Greengrass, le réalisateur, explique qu'il «développe l'histoire à mesure qu'[il] filme et avance. Ce qui veut dire qu'on doute jusqu'à la fin du processus/23 ». La productrice, Donna Langley, caractérise la méthode de Greengrass par sa volonté « d'improvisation, qui véhicule une impression d'immédiateté. Il jongle avec plusieurs balles en même temps, mais il sait toujours précisément où il va/24 ». Adopter une méthode de tournage plus libre, moins contraignante techniquement, permettrait donc de défendre une plus grande expression artistique.

\section{Les blockbusters d'auteur et le commerce de l'auteurisme}

Comme on le soulignait au début de cet article, on oppose traditionnellement les films d'auteur et les films à gros budget pour le grand public. Selon cette conception, l'auteurisme et les blockbusters hollywoodiens ne seraient pas compatibles. Afin de minimiser les risques financiers, le cinéma hollywoodien s'est réfugié dans des productions aux valeurs sûres comme les adaptations, les remakes, les sequels 
(les suites) ou encore les prequels/25. Aux yeux des producteurs, les réalisateurs et les scénaristes apparaissent interchangeables et ne sont pas des promesses de succès. Si récemment certains d'entre eux, comme J.J. Abrams (Star Trek, Super 8), M. Night Shyamalan (Le Sixième sens, Incassable, Signes), Peter Jackson (Le Seigneur des anneaux, King Kong), sont devenus des marques voire des plans marketing pour créer une nouvelle génération de réalisateurs vedettes qui succèderait à Steven Spielberg, Martin Scorsese, Georges Lucas, Tim Burton ou encore James Cameron, leur espace artistique reste limité et compliqué à négocier.

Cependant le succès grandissant des films indépendants incite les majors hollywoodiennes à reconsidérer la valeur ajoutée du réalisateur, et les pousse à engager directement des réalisateurs issus du secteur indépendant pour diriger des grosses productions (Christopher Nolan, Sam Raimi, Quentin Tarantino, Gus Van Sant, les frères Cohen, Steven Soderbergh, Michel Gondry). La récupération de ces auteurs vedettes indépendants permet ainsi de repenser, et à terme de légitimer, le commerce de l'auteurisme pour le grand public. Cette nouvelle labellisation de films à grand budget et de qualité, appelés désormais, les «blockbusters d'auteur », fonctionne comme un outil de distinction au sein même du cinéma dominant et grand public, de la même manière que le recyclage de l'amateurisme indépendant fonctionne comme un outil d'authentification. Empruntant ainsi le discours d'ordinaire associé aux réalisateurs de films indépendants, la promotion de ces blockbusters d'auteur s'axe principalement sur la légitimation d'un discours de l'authenticité et de la liberté artistique. Malgré leur récente reconversion dans un cinéma dominant qu'ils refusaient auparavant, les réalisateurs réaffirment sans cesse leur intégrité, en défendant leur position d'outsider et leur volonté d'exprimer une vision personnelle et alternative, effaçant ainsi les différences avec leurs discours précédemment défendus lorsqu'ils travaillaient encore dans le secteur indépendant. Il est intéressant de noter les rapprochements entre les discours de deux figures de proue des deux secteurs, Jim Jarmusch et M. Night Shyamalan.

Connu pour son franc parler et son statut d'outsider intègre, le réalisateur indépendant Jim Jarmusch n'hésite pas à critiquer son propre camp (le secteur indépendant) pour renforcer son indépendance. À propos de la labellisation même du terme « indépendant », il déclare volontiers à un journaliste du Guardian: "On ne parle que des indépendants. J'en ai marre de ce mot. J'ai envie de prendre mon revolver à chaque fois que je l'entends. [...] Ces mots sont devenus des labels qui sont collés sur des produits pour les vendre. Tout ceux qui font des films, qui ne sont pas définis par des concepts marketing ou des
/25 « Euvre réalisée après une œuvre de référence, mais dont l'action se déroule précédemment d'un point de vue scénaristique », source Wipidépia [http:// fr.wikipedia.org/wiki/ Pr\%C3\%Agquelle]. 
/ 26 Lynn Hirschberg, «Popular Success is Not My Area ", The Guardian, 5 août 2005, [http://www. guardian.co.uk/ film/2005/aug/05/2?INT CMP =ILCNETTXT34 87].

\section{/27 Sean O'Hagan, «Wandering Star », The Guardian, 2 octobre 2005, [http://www. guardian.co.uk/film/ 2005/oct/02/features. magazine].}

/ 28 Richard Corliss, « M. Night Shyamalan's Scary Feature », Time, 8 juin 2008, [http://www. time.com/time/magazine/ article/o,9171,1214952,00 .html].

/29 Richard Corliss, op. cit.

/30 Allison Hope Weiner, "Shyamalan's Hollywood Horror Story, With Twist », New York Times, 2 juin 2008, [http://www. nytimes.com/2008/06/ 02/business/media/02 night.html].

/31 Roger Ebert, " The Happening: Movie Review ", rogerebert.com, 13 juin 2008, [http:// rogerebert.suntimes.com/ apps/pbcs.dll/ article?AID=/20020802/ REVIEWS/208020305/ 1023]. entreprises commerciales, sont indépendants. Mes films sont faits à la main. Ils ne sont pas polis, ils sortent en quelque sorte de mon garage. C'est comme si j'étais un artisan en quelque sorte/26. ». L'énervement du réalisateur sous-entend son refus d'appartenir non seulement à Hollywood mais également à l'indiewood. Renforçant son opposition à ces deux systèmes institutionnalisés, il déclare dans un second entretien au Guardian: "Pour moi, la politique des auteurs, c'est des conneries. [...] Je ne veux pas être mainstream. J'aime être à la marge. Je suis content d'y être. Les choses qui m'inspirent sont à la marge. Je n'essaie pas d'être consciemment marginal, c'est juste ma manière de fonctionner et de vivre/27. ".

Dans le secteur hollywoodien, M. Night Shyamalan est l'auteur de nombreux films à succès dont Le Sixième Sens (1999), Incassable (2000) et plus récemment Phénomènes (2008). II aime à rappeler lui-même qu'il est une valeur sûre pour l'économie hollywoodienne: «Excepté Pixar, j'ai réalisé coup sur coup les quatre films les plus originaux de tous les temps. [...] Si vous voulez parier sur moi, alors tout le monde devrait en avoir pour son argent. J'ai transformé le profit en principe mathématique imparable. Je suis le pari le plus sûr que vous ayez sur le marché/28. ». Champion du box-office américain, Shyamalan s'est cependant fait une réputation d'outsider, au même titre que Jarmusch. Évitant les interviews avec la presse, refusant de négocier avec des producteurs belliqueux, en 2005 il va jusqu'à claquer la porte des studios Disney, qui avaient pourtant produit ses quatre premiers succès hollywoodiens. Comme l'explique Michael Bamberger dans son ouvrage, The Man Who Heard Voices: Or How M. Night Shyamalan Risked His Career on a Fairy Tale, le studio lui aurait pourtant offert 60 millions de dollars pour produire La Jeune fille de l'eau (2006) mais le réalisateur aurait estimé que les producteurs n'aimaient pas véritablement son film. « Les éléments qui me rendaient conventionnel étaient appréciés, et les autres qui me rendaient non conventionnels ne l'étaient pas. Je savais qu'une grande partie de mon travail n'était pas conventionnel et je ne voulais pas abandonner cette partie de moi/29 ", déclare-t-il au New York Times quelques années plus tard pour expliquer sa décision. En effet, le réalisateur est persuadé qu'il doit son succès non pas à sa fidélité à un studio mais au contraire à son anticonformisme et à son refus des normes hollywoodiennes: « Je ne serais pas là où je suis maintenant si je n'avais pas refusé ces conventions depuis le début/30. ». Ce refus des conventions est d'ailleurs reconnu dans le discours critique également. Ainsi Roger Ebert écrit à propos de Phénomènes: «À une époque où Hollywood confond volume et action, Shyamalan fait des films silencieux. À une époque où l'action fait le style, il nous persuade de faire attention aux moindres nuances./31 ». 
Bien que ces deux exemples se rapprochent en termes de discours, Jarmusch et Shyamalan sont néanmoins deux réalisateurs qui n'ont jamais changé de secteur. Si l'on s'intéresse à un réalisateur comme Christopher Nolan, il est intéressant de noter l'évolution de son discours. Nolan s'est fait remarqué d'abord par ses films indépendants, notamment Memento (2000). Mais très rapidement repéré par Hollywood, il s'est lancé dans des films à plus gros budget dont les deux volets de Batman (Batman Begins, 2005; The Dark Knight, 2008) et plus récemment Inception (2010). Il représente cette nouvelle catégorie d'auteur, d'abord indépendants puis hollywoodiens, que Yannis Tzioumakis nomme des « auteurs industriels/32 ». Cette labellisation présuppose que le réalisateur soit déjà considéré comme un artiste avant le succès qu'il peut rencontrer à Hollywood, ce qui permet de vendre ses films sur la base de son nom et de sa reconnaissance critique et publique déjà préexistante. «C'est cette association [...] qui permet au réalisateur de conserver sa réputation auprès de spectateurs de plus en plus exigeants/33. ". C'est ainsi que Nolan a pu être un auteur commercial, dont la sensibilité, l'intelligence et la créativité sont régulièrement saluées. Paradoxalement, il ne tient pas le discours de Shyamalan visant à changer le système de l'intérieur, d'user de sa position d'outsider indépendant. Au contraire, Nolan soutient sans complexe en entretien qu'il « ne se considère pas du tout comme un réalisateur de films d'art », préférant même avouer : « en réalité, j'ai des goûts mainstream, même si ça peut paraître surprenant/34 ». Nolan, contrairement à la tendance majoritaire, ne tente pas de minimiser ses budgets. Selon lui, le succès commercial ne compromet pas la vision personnelle d'un réalisateur, au contraire « un budget réduit vous compromet! Avec un micro-budget, vous êtes constamment en train de vous convaincre que les compromis que vous faites ne sont pas des sacrifices artistiques, et avec un gros budget, vous êtes constamment en train de vous convaincre que les compromis que vous faites en accord avec les financeurs, sont appropriés et constructifs/35 ». Christopher Nolan défend ainsi ardemment une position inverse, selon laquelle le budget permettrait de ne faire aucun compromis et sacrifice sur sa vision originale et qu'en ce sens, ce serait un outil de libération.

Finalement la mise en avant du réalisateur comme gage de qualité permet de repenser l'usage du discours de l'auteurisme dans le discours critique et promotionnel des blockbusters d'auteur, ainsi que de repenser la construction de la persona de ces auteurs vedettes. Le rapprochement entre deux secteurs d'ordinaire strictement opposés propose une réconciliation possible entre deux publics tout autant opposés, le public cinéphile et spécialiste, ainsi que le grand public,
/32 Yannis Tzioumakis, «Marketing David Mamet: Institutionally Assigned Film Authorship in Contemporary American Cinema ", The Velvet Light Trap, printemps 2006, $\mathrm{n}^{\circ} 57$.

/33 ibid., p. 72.

/34 Chris Roberts, «Memento More-ish: Interview with Christopher Nolan ", The Unofficial Christopher Nolan Website, 17 octobre 2000, en ligne [http://www. christophernolan.net/ interviews_m_virgin.php].

/35 ibid. 
rapprochement qui est également manifeste dans l'étude de réception des blockbusters d'auteur.

Dépossédé de ce qui le caractérisait en premier lieu, le cinéma indépendant américain entretient des rapports de plus en plus complexes avec Hollywood. Les définitions esthétiques ou économico-industrielles ne suffisent plus. Les discours de l'authenticité, de la sincérité et de l'intégrité artistique sont aujourd'hui tenus autant par des réalisateurs fidèles au secteur indépendant, que par ceux qui ont intégré Hollywood. Plus qu'une catégorie industrielle et économique, le cinéma indépendant américain apparaît donc aujourd'hui comme une idée productive et compétitive - le discours d'opposition à Hollywood est encore minoritaire au sein des réalisateurs commerciaux. Une nouvelle approche mérite d'être envisagée pour définir le secteur. Nous proposons qu'il soit alors compris plutôt comme un mode de consommation et d'appréciation culturel, bien différent de son équivalent hollywoodien. Le cinéma indépendant agit moins en opposition à Hollywood, moins comme une alternative, que comme un processus de différenciation symbolique, un processus de hiérarchisation, une culture de goût. À partir des observations et des conclusions tirées de cet article, la nouvelle problématique qui se dégage serait la suivante: le terme de « cinéma indépendant américain » aurait-il évolué d'un mode de production et d'un projet esthétique vers un label culturel et discursif qui connoterait un nouveau mode de consommation et d'appréciation culturelle?

\section{Célia Sauvage}

